\title{
Asthma: where is it going?
}

\author{
D.S. Faffe
}

Laboratório de Fisiologia da Respiração, Instituto de Biofísica Carlos Chagas Filho, Universidade Federal do Rio de Janeiro, Rio de Janeiro, RJ, Brasil

Correspondence to: D.S. Faffe, Laboratório de Fisiologia da Respiração, Instituto de Biofísica Carlos Chagas Filho, Universidade Federal do Rio de Janeiro, Centro de Ciências da Saúde, Bloco G, Sala G2042, Av. Carlos Chagas Filho, s/n, 21941-902 Rio de Janeiro, RJ, Brasil

Fax: +55-21-2280-8193. E-mail: faffe@biof.ufrj.br

\begin{abstract}
Asthma is characterized by reversible airway obstruction, airway hyperresponsiveness, and airway inflammation. Although our understanding of its pathophysiological mechanisms continues to evolve, the relative contributions of airway hyperresponsiveness and inflammation are still debated. The first mechanism identified as important for asthma was bronchial hyperresponsiveness. In a second step, asthma was recognized also as an inflammatory disease, with chronic inflammation inducing structural changes or remodeling. However, persistence of airway dysfunction despite inflammatory control is observed in chronic severe asthma of both adults and children. More recently, a potential role for epithelial-mesenchymal communication or transition is emerging, with epithelial injury often resulting in a self-sustaining phenotype of wound repair modulation by activation/ reactivation of the epithelial-mesenchymal trophic unit, suggesting that chronic asthma can be more than an inflammatory disease. It is noteworthy that the gene-environmental interactions critical for the development of a full asthma phenotype involve processes similar to those occurring in branching morphogenesis. In addition, a central role for airway smooth muscle in the pathogenesis of the disease has been explored, highlighting its secretory function as well as different intrinsic properties compared to normal subjects. These new concepts can potentially shed light on the mechanisms underlying some asthma phenotypes and improve our understanding of the disease in terms of the therapeutic strategies to be applied. How we understand asthma and its mechanisms along time will be the focus of this overview.
\end{abstract}

Key words: Asthma; Epithelial-mesenchymal unit; Bronchial hyperresponsiveness; Remodeling

Received July 19, 2007. Accepted July 1, 2008

\section{Introduction}

Asthma represents a serious public health problem throughout the world, and its prevalence is increasing both in developed and developing countries (1). Despite significant advances in our understanding of the disease and availability of therapies, limitations still persist: some patients do not respond or stop responding to corticosteroid treatment (2), long-term loss of lung function in asthmatic subjects persists despite adequate anti-inflammatory therapy (3), and airway structural changes and remodeling poorly improve with therapy. This overview concentrates on how the understanding of asthma and its mechanisms has been evolving with time.

Asthma is characterized by reversible airway obstruction, airway hyperresponsiveness, and airway inflammation, mainly with eosinophil and Th2 lymphocyte infiltration. The first mechanism identified as important for asthma pathophysiology was bronchial hyperresponsiveness $(\mathrm{BHR})$, and the disease was understood as a disorder of the conducting airways, which contracted too much and too easily. In a second step, asthma was recognized also as an inflammatory disease. The inflammatory process was identified as a major component of the disorder, where acute and subacute inflammatory responses superimposed on chronic inflammation, leading to structural changes and airway remodeling. More recently a new concept is emerging which may probably help to understand what is going on in chronic asthma, which seems to be more than an inflammatory disease. Although most asthma cases can be satisfactorily managed with a combination of anti-inflammatory drugs and bronchodilators, patients who remain symptomatic despite maximum combination treatment represent a heterogeneous group, in which a subphenotype can be characterized by heightened neutrophilic airway inflammatory response in the presence or 
absence of eosinophils, with evidence of increased tissue injury and remodeling. Aberrant communication between an injured airway epithelium and underlying mesenchyme contributes to disease chronicity and refractoriness to corticosteroids (4). Many patients with severe asthma show persistent airway dysfunction despite inflammatory control (5) and anti-inflammatory therapy does not improve airway remodeling. In fact, airway remodeling has been detected in children with short-term underlying airway inflammation $(6,7)$. Taken together, these observations suggested the hypothesis of a role for activation or reactivation of the epithelial-mesenchymal unit, involving mechanisms similar to those observed during normal lung morphogenesis.

\section{Bronchial hyperresponsiveness}

Bronchial hyperresponsiveness, excessive airway narrowing caused by stimuli that normally elicit a limited or no response, has been recognized as a prominent feature of asthma, and is manifested by a combination of increased sensitivity and reactivity to a given stimulus (8). Although BHR broadly correlates with asthma severity, a cause and effect relationship is far from clear. The understanding of this mechanism is limited by experimental difficulties. It is noteworthy that the BHR measured in the laboratory, with direct stimulation of the airway smooth muscle by the use of bronchial provocation drugs, differs from that elicited by environmental stimuli (e.g., air pollutants, allergens, cold air), which induces airway narrowing indirectly through the release of mediators (9). Acute narrowing of the airway lumen is caused by contraction of the airway smooth muscle (ASM) cell, but a causal link has been equivocal (10). Although ASM behavior can be modified by the presence of inflammation in the local environment $(11,12)$, the airway inflammation in asthmatic patients does not correlate with the degree of airway responsiveness $(5,8)$. There is great interest in understanding if the airway muscle per se differs between asthmatic and non-asthmatic subjects, or if it simply responds to an altered environment.

Functional changes have been reported in the ASM behavior of asthmatic patients, suggesting that the intrinsic contractile properties of ASM are altered in asthma $(8,13,14)$. Increased response to contractile agonists $(15,16)$ and reduced response to dilator agonists have been well documented $(11,17)$. The oldest and simplest explanation of why the muscle can shorten excessively in asthma is that muscle from the asthmatic airways is stronger than muscle from non-asthmatic airways, but evidence for this hypothesis is equivocal (18). On the other hand, the alternative hypotheses were consistent with remodeling events induced by an inflammatory microenvironment, e.g., changes of muscle mass, changes of the static load against which the muscle shortens, and changes of the dynamic load of myosin binding. In humans, however, bronchospasm has been reported in the absence of acute inflammation in exercise-induced asthma, with no significant increase in bronchoalveolar lavage levels of inflammatory mediators and/or cells (14). Indeed, the extent and functional implications of the various elements present during remodeling are uncertain (8).

The first evidence for an intrinsic abnormality of ASM cells came from the observation that ASM cells from patients with asthma proliferate significantly faster than cells from control subjects (19). Indeed, a recent report demonstrated reduction of asthma symptoms and improved lung function after removal of ASM bundles (20). Other studies, however, have reported increased ASM contractility with no change in ASM mass in in vitro and in vivo models of airway hyperresponsiveness $(21,22)$.

In addition, increased velocity of muscle shortening has been demonstrated in animal models of airway hyperresponsiveness $(21,23)$, and in human ASM from asthmatic patients, in which these functional differences were associated with an increased message for myosin light chain kinase (14). ASM velocity of shortening plays a significant role in determining the maximal extent of smooth muscle shortening for a given load (22), supporting its relevance to airway hyperresponsiveness (8). These findings suggest that the cell itself seems to be mechanically different, supporting the argument that ASM cells are the seat of primary changes in the mechanical properties of the asthmatic airways (14).

Other reports indicate that ASM cells from patients with asthma have an activated phenotype that may be attributed to an intrinsic or acquired abnormality. ASM cells from these patients produce more connective tissue growth factor, an important contributor to extracellular matrix deposition, in response to transforming growth factor- $ß$ (TGF- $ß$ ) (24), while they produce less prostaglandin E2 as a result of lower cyclooxygenase-2 expression, which could result in less airway relaxation or lower the threshold for ASM cell contraction (25).

Another difference between ASM from asthmatics and non-asthmatics is related to its response to deep inspirations. Previous studies have reported bronchodilation and a bronchoprotective effect of deep inspiration on the airways, which was absent or diminished in asthma (26). Indeed, in asthmatics, deep inspiration fails to reverse spontaneous obstruction, and sometimes even results in worsening of airway narrowing (27-29). Furthermore, the degree of bronchoconstriction induced by deep inspiration is related to spontaneous obstruction severity $(28,29)$. 
Two hypotheses have been raised in an attempt to explain the mechanism by which lung inflation confers protection on the non-asthmatic lung. The first is a direct effect of stretching on ASM. The depression of contractility during length oscillations is explained as a function of the plasticity of contractile filaments within ASM cells, which allows contractile element length to be reset in relation to smooth muscle cell length as a result of changes in the length to which the muscle is stretched (30), or of changes in the cycling and nature of the myosin cross bridges (31). The second hypothesis is that airway stretch activates a process that antagonizes a bronchoconstrictive stimulus in a functional way (26). However, the question remains as to why the effects of deep inflation are absent in asthma. These effects may become defective because lung inflation may not be capable of stretching the airway, because the airway may be stretched but its effect on ASM is defunctionalized by airway inflammation, or because an intrinsic abnormality of the ASM prevents the bronchoprotective effect of airway stretch. Recent observations on ASM cells from naturally hyperresponsive and hyporesponsive rats support the idea that intrinsic abnormality of the ASM may interfere with the protective effects of lung inflation on asthmatic airways (13).

In addition, it is thought that the ability of the ASM cell to accommodate its cytoskeleton to progressively shorter working muscle lengths is a major factor contributing to the excessive airway narrowing in asthma (10). Recently, it has been demonstrated that ASM cells from the relatively hyperresponsive Fisher rat possess a higher content of ATP and higher effective temperature of its cytoskeleton lattice than the hyporesponsive Lewis strain, indicating that the biophysical properties of the ASM cell can contribute to the pathophysiology of BHR in asthma (10). Thus, although inflammatory mediators can affect ASM cell behavior (32), these new lines of evidence propose a supportive mechanism for BHR, which is independent of inflammation or remodeling.

\section{Airway inflammation}

Asthma is recognized as a chronic inflammatory disorder, whereas airway inflammation is a constant feature within a highly variable clinical spectrum, and is persistent even though symptoms are episodic (33). The inflammatory process is dominated by Th2 cells, which release specific cytokines including interleukin (IL)-4, IL-5, IL-9, and IL-13, that orchestrate eosinophilic inflammation and $\lg \mathrm{E}$ production by $\mathrm{B}$ lymphocytes. Although eosinophils and $T$ cells have received substantial attention, the interactions among multiple cell types, including basophils, mast cells, epithelial cells, macrophages, and B lymphocytes, add further complexity to the pathogenesis of asthma. Structural cells of the airways also produce inflammatory mediators and contribute to the persistence and amplification of the inflammatory process (Table 1) $(32,34)$. Th2 cells are involved mainly in allergic disorders, and are responsible for the humoral response. In contrast, Th1 cells produce interferon- $\gamma$ and IL-12, and promote the cell immune response. In asthma, the balance between Th1 and Th2 cytokine production is more important than the absolute levels of individual cytokines (34). Th1 and Th2 cells differentiate from a common precursor in response to the local environment and to regulatory cells.

Over 100 different mediators are now recognized to be involved in asthma and to mediate the complex inflammatory response of the airways by activating different signaling pathways and transcription factors. Cytokines orchestrate the inflammatory response and determine disease severity (Figure 1). Key cytokines involved in asthma pathophysiology include IL-1ß and TNF- $\alpha$, which amplify the inflammatory response through activation of the transcription factors NF- $\mathrm{KB}$ and $\mathrm{GM}-\mathrm{CSF}$, which prolong eosinophil survival, and the Th2-derived cytokines: IL-5, required for eosinophil differentiation and survival, IL-4, important for Th2 cell differentiation, and IL-13, required for IgE formation and directly related to BHR. The main Th2 cytokines, IL-4 and IL-13, act by activating the signal transducer and activator of transcription 6 (STAT-6) pathway, and also MAP kinase in some cell type, such as ASM cells $(32,35)$. The release of chemokines is also important in the recruitment of inflammatory cells into the airways, including eotaxin, which is relatively selective for eosinophils; thymus and activation-regulated chemokine (TARC) and MDC, which recruit Th2 cells; RANTES, a chemoattractant for eosinophils and lymphocytes secreted by epithelial and $T$ cells; monocyte (MCP-1 and MCP-2) and eosinophil (MCP3 and $\mathrm{MCP}-4)$ chemoattractant released by macrophages (macrophage inflammatory protein, MCP-1), T cells (MCP2, -3), epithelial (MCP-1, -4) and smooth muscle cells (MCP-1), and MIP-1 1 , chemoattractant for eosinophils and basophils, released by eosinophils, basophils, neutrophils, and epithelial and smooth muscle cells $(34,35)$. Chemokines are expressed mainly in airway epithelial cells; however, recent reports also highlighted a key role for ASM cells in chemokine release, including eotaxin and $\operatorname{TARC}(32,35,36)$.

A relationship between asthma severity and the intensity of inflammation has not been clearly established. Although studies on both asthmatic adults and children have reported dissociation between airway inflammation and the degree of airway responsiveness $(5-7,37,38)$, 
Table 1. Cells involved in the pathogenesis of asthma.

\begin{tabular}{|c|c|c|}
\hline Cell type & Function & Released products \\
\hline \multicolumn{3}{|l|}{ Inflammatory cells } \\
\hline Mast cells & Linked to bronchial hyperresponsiveness & $\begin{array}{l}\text { Bronchoconstrictor mediators: histamine, } \\
\text { leukotrienes, PGD2 }\end{array}$ \\
\hline Eosinophils & Role in release of growth factor and remodeling & Cytotoxic mediators: MBP, ECP, EPO \\
\hline T lymphocytes & $\begin{array}{l}\text { Orchestrate eosinophilic inflammation and IgE } \\
\text { production }\end{array}$ & IL-4, IL-5, IL-9, IL-13 \\
\hline Dendritic cells & $\begin{array}{l}\text { Sample allergens, interact with regulatory } T \text { cells, } \\
\text { stimulate production of Th2 cells }\end{array}$ & \\
\hline Macrophages & Activated by allergens & $\begin{array}{l}\text { Inflammatory mediators and cytokines that } \\
\text { amplify the inflammatory response, such } \\
\text { as IL-1ß and TNF- } \alpha\end{array}$ \\
\hline Neutrophils & $\begin{array}{l}\text { Increased in severe asthma and smoking } \\
\text { asthmatics, pathophysiological role is } \\
\text { uncertain. Secreted products recruit } \\
\text { additional inflammatory cells }\end{array}$ & $\begin{array}{l}\text { Proinflammatory: TNF- } \alpha, \text { IL-1, IL-6; } \\
\text { hematopoietic: IL-3, GM-CSF, G-CSF; } \\
\text { cytokines; chemokines: IL-8 }\end{array}$ \\
\hline B lymphocytes & Production of antigen-specific immunoglobulin & $\lg E$ \\
\hline Basophils & $\begin{array}{l}\text { Uncertain, probably implicated in late-onset } \\
\text { allergic response }\end{array}$ & IL-4, IL-13, MIP-1 $\alpha$, histamine, leukotriene \\
\hline \multicolumn{3}{|l|}{ Structural cells } \\
\hline Airway epithelial cells & Sense their mechanical environment & Cytokines, chemokines, and lipid mediators \\
\hline Airway smooth muscle cells & $\begin{array}{l}\text { Main effectors of bronchial hyperresponsiveness, } \\
\text { perpetuate and amplify inflammatory response, } \\
\text { participate in remodeling }\end{array}$ & $\begin{array}{l}\text { Cytokines: IL-4, IL-13, TNF- } \alpha \text {; chemokines: } \\
\text { eotaxin, TARC; growth factor: VEGF }\end{array}$ \\
\hline Endothelial cells & Role in recruiting inflammatory cells from circulation & \\
\hline Fibroblasts and myofibroblasts & Involved in airway remodeling & $\begin{array}{l}\text { Connective tissue components, such as } \\
\text { collagen and proteoglycans }\end{array}$ \\
\hline
\end{tabular}

PGD2 = prostaglandin D2; MBP = major basic protein; $\mathrm{ECP}=$ eosinophilic cationic protein; $\mathrm{EPO}=$ eosinophil peroxidase; $\mathrm{IL}=$ interleukin; TNF- $\alpha$ = tumor necrosis factor- $\alpha$; GM-CSF = granulocyte-macrophage colony-stimulating factor; G-CSF = granulocyte colony-stimulating factor; MIP-1 $\alpha$ : macrophage inflammatory protein-1 $\alpha$; TARC = thymus and activation-regulated chemokine; VEGF = vascular endothelial growth factor $(33,34,36,42)$.

Figure 1. Schematic representation of cytokine role in the response to different inflammatory diseases. Cytokines usually act through membrane receptors initiating intracellular signaling, with transcription factor activation, followed by nuclear stimulation, and the production of inflammatory mRNA and protein.

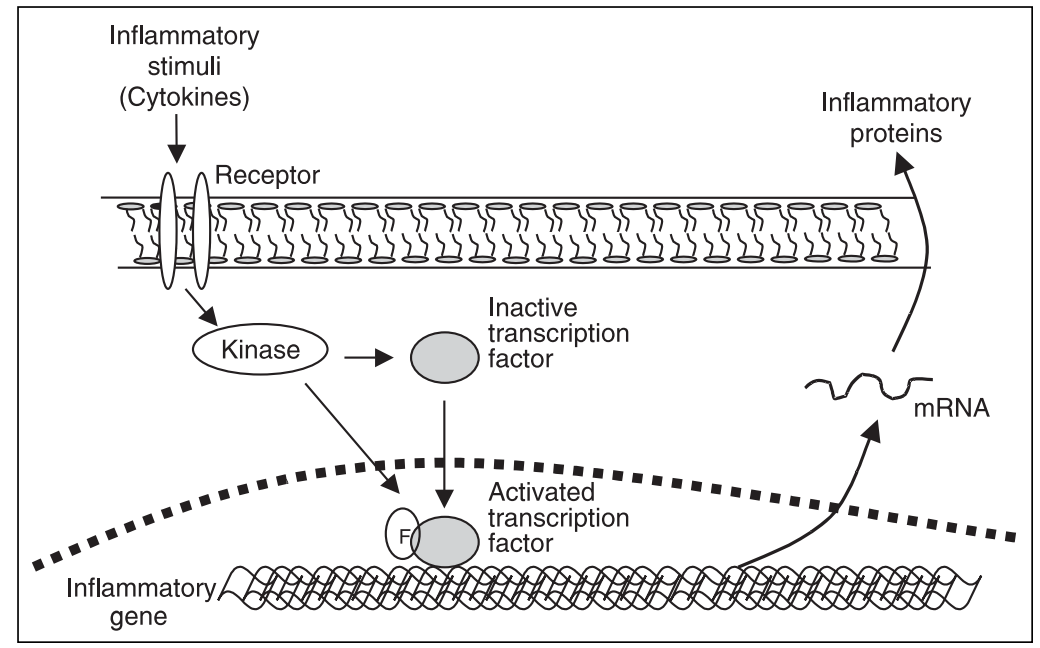


inflammation alone may lead to some features of asthma, such as reversible bronchospasm (39). In addition, chronic inflammation can cause tissue injury and structural changes, collectively referred to as remodeling. Airway remodeling is characterized by increased wall thickness, subepithelial fibrosis, smooth muscle hypertrophy and hyperplasia, epithelial mucus metaplasia, increased vascularity, and abnormalities in extracellular matrix composition (40). These structural changes may account for some characteristics of asthmatic physiology that are poorly addressed by current anti-inflammatory treatment (41). Airway wall thickness correlates with disease severity and length of time with disease, while smooth muscle thickness correlates with fatal versus nonfatal asthma (40). Airway remodeling has been proposed to explain aspects of asthma severity, such as airway hyperresponsiveness and fixed airway obstruction (41). Indeed, an animal model of airway remodeling suggests that airway dysfunction persists after resolution of inflammation, implicating airway remodeling as a factor in airway dysfunction (37). Remodeling is also considered to be related to the more rapid decline in lung function over time occurring in asthmatic subjects compared to nonasthmatics, a characteristic that has not been modified by corticosteroid treatment (3).

The traditional view that ASM is a purely contractile tissue in asthma, a "passive partner" responding to exogenous substances in an adverse environment, seems to be inadequate $(32,35)$. Compelling evidence now suggests that ASM plays an important role in regulating bronchomotor tone, in perpetuating airway inflammation, and in airway remodeling (32). Several publications have demonstrated that ASM, in addition to having contractile and proliferative properties, is also an important source of pro-inflammatory cytokines, chemokines and growth factors, as well as extracellular matrix components $(19,35,36$, 42,43).

Several interleukin molecules have a direct role in propagating the asthmatic inflammatory process, such as IL-13, IL-4, IL-5, IL-6, and IL-11 (32,35,36,38,39,42-44). A cycle beginning with Th2 inflammation and culminating in remodeling has been described. ASM cells are directly involved in this cycle, perpetuating lung inflammation (40). In addition, human ASM cells in culture can also produce several compounds implicated in remodeling, including fibronectin, laminin, percelan, chondroitin, and vascular endothelial growth factor $(42,43,45,46)$. This may be especially relevant in the presence of increased smooth muscle mass observed in asthmatic lungs (35).

Much effort has been made to characterize the diverse molecules secreted by ASM and to determine how they are induced. However, much less is known about the mechan- isms that regulate the secretory response of ASM compared with its proliferative mechanisms. Thus, several studies have begun to address the intracellular signals necessary for ASM secretory activity $(32,35)$. In ASM cells, IL-4 and IL-13 activate a STAT-6, as well as p38 and extracellular signal-regulated kinase pathways, leading to TARC and eotaxin release, respectively $(35,36)$. IL-1ß and TNF- $\alpha$ also initiate signaling in ASM cells. Through p38 and extracellular signal-regulated kinase activation, IL-1ß induces cytokine and chemokine release by ASM cells, including eotaxin, RANTES, and GM-CSF; while TNF- $\alpha$ has been demonstrated to induce IL-6, ICAM-1, and RANTES by pathways involving NF- $\kappa B$ and AP1 activation $(32,35)$. These cytokines involved in the asthma phenotype also synergize with oncostatin M (OSM), which activates the STAT-3 pathway in ASM culture, increasing MCP-1, IL-6 and eotaxin release. Moreover, ASM cells in culture can also release growth factors such as vascular endothelial growth factor after IL-4, IL-13 and OSM stimulation, an effect strongly enhanced by the presence of OSM together with IL-4/IL-13 or with IL-1ß. These findings suggest a role for ASM cells in airway remodeling $(42,43)$.

\section{Epithelial-mesenchymal unit}

Persistence of airway dysfunction despite inflammatory control is observed in chronic severe asthma in both adults and children $(3,47)$. More recently, a potential role for the epithelial-mesenchymal communication or transition has been emerging, which leads to the idea of chronic asthma as more than an inflammatory disease.

Remodeling was classically understood as a direct consequence of inflammation, which means that it depended on chronic and sustained inflammation. However, structural changes poorly improve with anti-inflammatory therapy. Furthermore, subepithelial fibrosis, a marker for remodeling, is actually also a very early marker for the asthmatic phenotype in young children, whereas it does not correlate with duration of the disease or necessarily with the severity of inflammation $(6,7)$. Taken together, these observations suggest that some features of asthma physiology may be related to mechanisms other than chronic inflammation. Two parallel events have been proposed as necessary for asthma to become chronic and persistent: a susceptible airway linked to altered structure and function, and a microenvironment capable of sustaining a chronic inflammatory response (9). Recent observations suggest that abnormal tissue injury and repair would provide this appropriate microenvironment for persistent inflammation and remodeling (48). Studies with bronchial biopsies suggest that, in asthma, the airway epithelium is 
more susceptible to injury than the normal epithelium $(49,50)$. Indeed, epidermal growth factor receptor (EGFR) and the epithelium isoform of CD44 expression, markers of epithelial injury in vivo, increase in asthma in proportion to disease severity, and throughout the epithelium, suggesting a widespread damage. Also, epithelial expression of the neutrophil chemoattractants IL- 8 and MIP- $1 \alpha$ is increased in severe asthma and correlates with increased EGFR expression. Thus, the effect of different components of the inhaled environment, such as oxidants, on a susceptible epithelium can trigger epithelium activation and damage in asthma (49). Furthermore, in chronic asthma the airway epithelium seems to be impaired in its ability to reconstitute itself after injury, entering into a chronic wound response, with increased secretion of profibrogenic growth factors, such as fibroblast growth factor (FGF)-2. Actually,
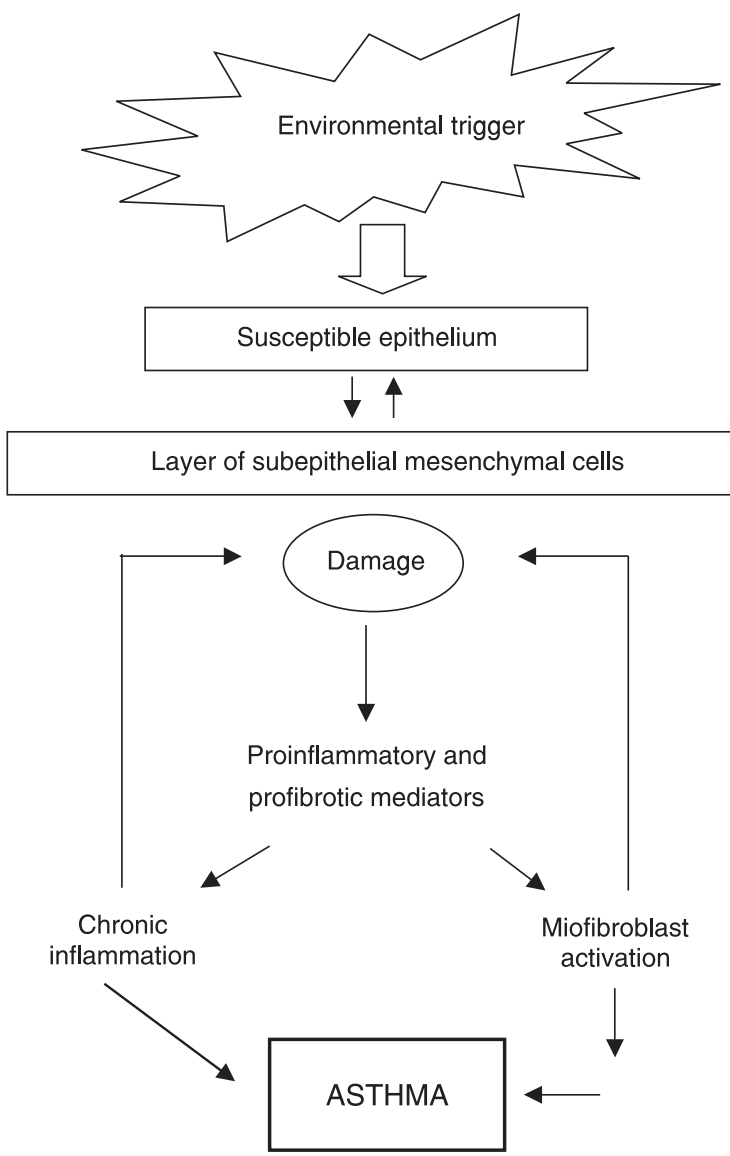

Figure 2. Epithelial-mesenchymal communication in asthmatic airways. The susceptible epithelium can release proinflammatory and profibrotic mediators that mediate remodeling and perpetuate the chronic inflammatory cycle. It is postulated that remodeling and inflammation attenuate the normal repair of damaged epithelium, leading to the development of asthma. a number of factors contribute to an ideal microenvironment for the persistence of Th2-mediated insulin-like growth factor (IGF)-1, and TGF-ß $(9,51)$, such as inflammation, including an epithelium more susceptible to injury, delayed epithelium repair, and an altered phenotype with the generation of growth factors that drive mesenchymal cell proliferation and differentiation towards increased matrix deposition and smooth muscle (9).

Data exist suggesting that epithelial damage participates in the pro-inflammatory process by releasing cytokines, growth factors, and mediators $(40,52)$. Epithelial damage induces up-regulation of receptors, specifically members of the EGFR family that drive proliferation and repair. Indeed, biopsies from asthmatic subjects demonstrate a marked increase in EGFR expression, which increases in proportion to disease severity and chronicity irrespective of corticosteroid treatment (9). Injury to epithelial monolayers in vitro results in increased release of fibroproliferative and FGF including FGF-2, IGF-1, platelet-derived growth factor, endothelin-1, and TGF- $ß$ as well as an array of EGFR ligands. TGF- $ß, F G F-2$ and endothelin-1 have been demonstrated to be increased in asthma, while both epithelial EGFR expression and TGF-ß production are refractory to corticosteroid treatment. This suggests that the combined effects of these signaling pathways on the epithelial mesenchymal trophic unit (EMTU) could be involved in tissue remodeling, explaining the incomplete resolution of lung function with anti-inflammatory treatment in chronic asthma (9,53-55). In severe asthma, however, a high level of EGFR expression is not followed by increased proliferation. Actually, an increase in p2 1 waf is demonstrable throughout the epithelium, where it impairs proliferation by arresting cells at the G1 phase of the cell cycle. Thus, in chronic asthma the airway epithelium seems to be impaired in its ability to reconstitute itself after injury, entering into a chronic wound response. The relationship between EGFR signaling and the repair and remodeling processes has been defined using EGFRselective tyrosine kinase inhibitors, suggesting that parallel pathways operate in repairing epithelial injury, some leading to reconstitution and regulated by EGFR, whereas others induce profibrogenic factor production independent of EGFR $(9,53)$. Thus, epithelial injury can result in a selfsustaining phenotype of wound repair modulation by activation/reactivation of the EMTU (Figure 2) (53-55). Collagen, reticular and elastic fibers, proteoglycans and glycoproteins, all of which contribute to lung remodeling, can be produced by mesenchymal cells influenced by epithelial cells. Thus, epithelial-mesenchymal interactions play an important role in extracellular matrix production and deposition $(52,53)$. Furthermore, the Th2-type cytokines IL-4 
and IL-13, signaling via the transcription factor STAT-6, are able to induce myofibroblast transformation and submucosal remodeling through a TGF-ß-depending mechanism. These cytokines also have direct proinflammatory effects on epithelial cells and fibroblasts, thus contributing to chronic inflammation and airway remodeling through their interaction with the EMTU (9).

The presence of remodeling features in bronchial biopsy specimens from young children in the absence of eosinophilic infiltrates suggests an early role for epithelialmesenchymal trophic interactions, secondary to epithelial injury and stress $(6,7)$. Actually, remodeling can be defined as structural changes during growth or in response to injury or inflammation. Airway remodeling is not an inherently abnormal response, but either an appropriate step in normal lung development or repair, or a series of events that can lead to pathophysiological consequences (52). Communication between the epithelium and the fibroblast sheet is reminiscent of the morphogenesis process during which epithelium and mesenchyme form a trophic unit (56). It is noteworthy that the gene-environmental interactions critical for the development of a full asthma phenotype involve processes similar to those occurring in branching morphogenesis $(9,55)$. The importance of the epithelial-mesenchymal interactions in branching morphogenesis and alveolus formation has been known for several decades. Although it was initially suggested that the instructive signaling was unidirectional, with the pulmonary mesenchyme producing signaling molecules such as NF- $\kappa \mathrm{B}$, TGF- $ß$ and FGF to induce normal epithelial development, it is now known that the epithelium also produces signaling molecules such as EGF, TGF-ß, Sonic Hedgehog (Shh) and Wnt proteins that are important for normal mesenchymal differentiation and proliferation (57). Moreover, the majority of transcription factors, growth factors and other signaling molecules involved in branching morphogenesis are also implicated in airway remodeling $(53,57)$. The observation that overexpression or inhibition of key elements of the EMTU results in dysfunctional repair has led to the proposal that remodeling in asthma could be a result of the EMTU remaining active after birth or becoming reactivated in susceptible asthmatics (57).

Some investigators propose that in chronic asthma the epithelial mesenchymal trophic unit, crucial to lung development, becomes reactivated, leading to pathologic remodeling $(9,57)$, whereby airway mesenchymal cells differentiate into myofibroblasts, which have both contractile and secretory functions $(58,59)$. Remodeling of the asthmatic airway is associated with accumulation and activation of fibroblasts immediately beneath the reticular basement membrane (57). TGF- $§$ and the related bone mor- phogenic proteins secreted by the epithelium and by inflammatory cells are powerful stimuli for the differentiation of airway mesenchymal cells into myofibroblasts. Although the relationship between myofibroblasts and ASM cells has yet to be determined, it has been suggested that airway fibroblasts provide a continuous source of precursor cells to generate new smooth muscle. In addition, TGF$ß$ enhances the capacity of these cells to release a wide variety of additional growth factors and cytokines (9). However, the origin of the increased number of mesenchymal cells is unknown. One hypothesis is that the epithelialmesenchymal transition (EMT) might serve as a local source of interstitial fibroblasts (57). Epithelial cells can be converted to mesenchymal cells by a process known as EMT $(54,55,59)$. During this transition, epithelial cells undergo molecular reprogramming, developing a new set of biochemical instructions, with complex changes in their architecture and behavior $(55,57)$. The molecular mechanisms that regulate EMT are considerably overlapping with those that control cell adhesion, motility invasion, survival and differentiation, with participation of cytokines associated with proteolytic digestion of basement membranes upon which epithelial cells reside, including metalloproteinases, TGF- $ß$, EGF, IGF-2 and FGF-2 (54). Tissue culture studies have shown that several extracellular activators can trigger EMT (including components of the extracellular matrix, such as collagen, and soluble growth factors, such as TGF- $ß$ and FGF), that extensive crosstalk exists between the signaling pathways that activate and repress EMT, and that EMT-inducing signaling pathways have many common endpoints, including down-regulation of epithelial $(E)$-cadherin (which is expressed in epithelial cells but not in mesenchymal cells) and expression of EMT-associated genes (55). In this way, mature epithelia change their phenotype following morphogenic pressure from epithelial stress, such as inflammation or wounding, leading to fibroblast production and fibrogenesis (Table 2). While traditional studies of fibrosis have focused on the production of extracellular matrix, this recent information suggests that epithelia can contribute by creating new fibroblasts $(54,59)$. It is noteworthy that fibroblasts are conventionally accepted as a cell type of limited diversity. However, fibroblasts formed by EMT may express a profile of genes, or residual receptors or signaling pathways representative of their original mature epithelium, carrying a unique epithelial signature. Thus, fibroblasts of EMT origin can be as heterogeneous as the universe of epithelia, suggesting that therapies against fibrosis may need more specificity $(54,59)$. Finally, the distinction between epithelial-mesenchymal interaction and epithelial-mesenchymal transition should be underscored: while epithelial- 
mesenchymal interaction refers to paracrine crosstalk between tissue epithelia and stromal fibroblasts, epithelialmesenchymal transition is a variant of transdifferentiation (whereby differentiated cells change into other differentiated cells) and a mechanism for dispersing cells in embryos, forming fibroblasts in injured tissues, or initiating metastases in epithelial cancer (54).

Bronchial biopsy studies have shown thickening of the reticular basement membrane, evidence of airway remodeling, in children with difficult-to-control asthma unrelated to duration of symptoms, lung function or eosinophilic inflammation $(6,7)$. These findings suggest that disordered epithelial-mesenchymal signaling may be a key feature in the origin of asthma, raising the possibility that the mechanisms of chronic and severe asthma may be different from that of mild disease (9). It is known that during branching morphogenesis intermittent EGF and TGF- $ß$ dominance produces alternating linear and branching of the developing airways (Figure 3 ). A disorder of this process may account for the continued remodeling of the airways of subjects destined to develop asthma (9). Furthermore, evidence suggests that molecular pathways responsible for lung development are activated postnatally to regulate host defense and wound healing (57). Both morphogenic and pathological roles of TGF- $ß$ have been well documented, while lung TGF- $ß$ levels immediately after premature birth correlate with adverse pulmonary outcome. Indeed, transgenic mouse studies provide direct evidence for a functional linkage between inflammatory mediators and developmental pathways, whereas alveolar hypoplasia can be experimentally induced by overexpression of TNF- $\alpha$ and IL-6. Finally, inflammation activates

Table 2. Functional and phenotypic characteristics of epithelial and mesenchymal cells.

\begin{tabular}{ll}
\hline Epithelial cells & Mesenchymal cells \\
\hline Form layers & Do not form organized layer \\
Closely adjoined by specialized membrane structures & No polarization \\
Apical-basolateral polarization & Contact neighboring cells only focally \\
Are motile, can move & No association with a basal lamina \\
Do not detach and move away from their layer & Fibroblast-like morphology \\
Grow as clusters in culture, maintaining cell-cell adhesion with neighbors & Highly motile in culture \\
Express epithelial (E)-cadherin & Do not express E-cadherin \\
\hline
\end{tabular}

Source: Reference 55.

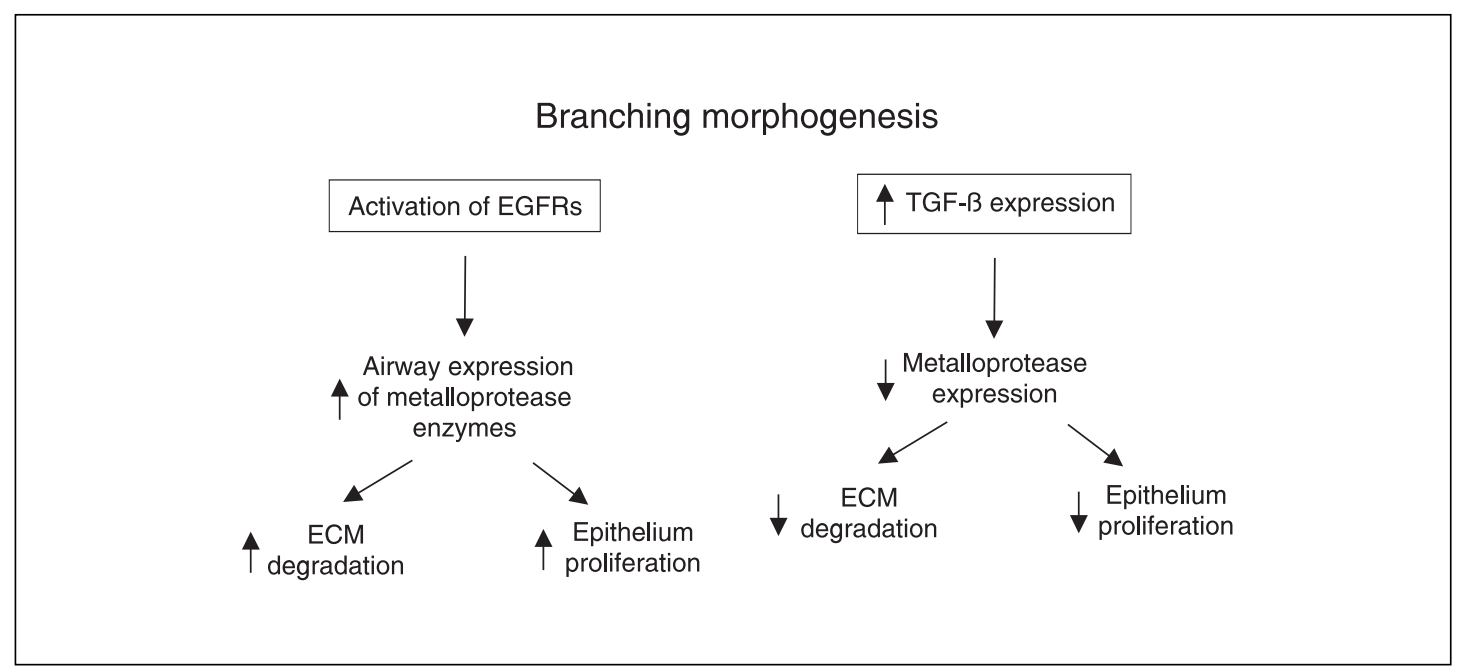

Figure 3. Communication between epithelium and the underlying epithelial fibroblast sheet is reminiscent of the process that drives branching morphogenesis, whereas epithelium and mesenchyme act as a trophic unit. Intermittent EGF and TGF- $\beta$ dominance produces alternating linear and branching of the developing airways, in a process similar to that observed in asthmatic airways (53). EGFRs $=$ epidermal growth factor receptors; TGF- $\beta=$ transforming growth factor- $\beta$; ECM = extracellular matrix. 
the ubiquitous transcription factor $\mathrm{NF}-\kappa \mathrm{B}$, which mediates many of its biological effects. On the other hand, perturbations of NF- $\kappa \mathrm{B}$ expression disrupt epithelial-mesenchymal interactions, resulting in abnormal lung morphogenesis. Thus NF- $\mathrm{KB}$ can modulate both inflammatory and morphological regulatory genes, establishing an operational and functional linkage between inflammation and development (60). Taken together, these data support a role for the reactivation of the epithelial-mesenchymal trophic unit as an important mechanism in chronic and severe asthma.

Although many different aspects in asthma pathophysiology have been increasingly elucidated with time, some limitations still persists in the management of asthmatic subjects. The recognition of asthma as an inflammatory disease and the description of different pathways involved

\section{References}

1. Eder W, Ege MJ, von Mutius E. The asthma epidemic. $N$ Engl J Med 2006; 355: 2226-2235.

2. Antonicelli L, Bucca C, Neri M, De Benedetto F, Sabbatani $\mathrm{P}$, Bonifazi $\mathrm{F}$, et al. Asthma severity and medical resource utilisation. Eur Respir J 2004; 23: 723-729.

3. Lange P, Parner J, Vestbo J, Schnohr P, Jensen G. A 15year follow-up study of ventilatory function in adults with asthma. N Engl J Med 1998; 339: 1194-1200.

4. Holgate ST, Polosa R. The mechanisms, diagnosis, and management of severe asthma in adults. Lancet 2006; 368: 780-793.

5. Crimi E, Spanevello A, Neri M, Ind PW, Rossi GA, Brusasco V. Dissociation between airway inflammation and airway hyperresponsiveness in allergic asthma. Am J Respir Crit Care Med 1998; 157: 4-9.

6. Fedorov IA, Wilson SJ, Davies DE, Holgate ST. Epithelial stress and structural remodelling in childhood asthma. Thorax 2005; 60: 389-394.

7. Payne DN, Rogers AV, Adelroth E, Bandi V, Guntupalli KK, Bush A, et al. Early thickening of the reticular basement membrane in children with difficult asthma. Am J Respir Crit Care Med 2003; 167: 78-82.

8. Gil FR, Lauzon AM. Smooth muscle molecular mechanics in airway hyperresponsiveness and asthma. Can J Physiol Pharmacol 2007; 85: 133-140.

9. Holgate ST, Holloway J, Wilson S, Bucchieri F, Puddicombe $S$, Davies DE. Epithelial-mesenchymal communication in the pathogenesis of chronic asthma. Proc Am Thorac Soc 2004; 1: 93-98.

10. An SS, Fabry B, Trepat X, Wang N, Fredberg JJ. Do biophysical properties of the airway smooth muscle in culture predict airway hyperresponsiveness? Am J Respir Cell Mol Biol 2006; 35: 55-64.

11. Jiang H, Rao K, Halayko AJ, Kepron W, Stephens NL. Bronchial smooth muscle mechanics of a canine model of allergic airway hyperresponsiveness. J Appl Physiol 1992; 72: $39-45$. in its inflammatory process and remodeling have greatly improved our understanding of its mechanisms. The latest evidence now reveals additional explanations and complexity for BHR, besides inflammation and remodeling. The concept of epithelial mesenchymal activation-reactivation together with the new findings on asthmatic ASM properties can potentially improve our understanding of the decline in lung function observed over time in this disease, as well as the anti-inflammatory refractory component of bronchial hyperresponsiveness, opening new avenues for therapeutic strategies in this disease. Finally, these findings also suggest that we have much more to learn about lung development that could shed new light on asthma pathophysiology.

12. Fernandes DJ, Mitchell RW, Lakser O, Dowell M, Stewart AG, Solway J. Do inflammatory mediators influence the contribution of airway smooth muscle contraction to airway hyperresponsiveness in asthma? J Appl Physiol 2003; 95: 844-853.

13. An SS, Bai TR, Bates JH, Black JL, Brown RH, Brusasco V, et al. Airway smooth muscle dynamics: a common pathway of airway obstruction in asthma. Eur Respir J 2007; 29: 834860.

14. Ma X, Cheng Z, Kong H, Wang $\mathrm{Y}$, Unruh H, Stephens NL, et al. Changes in biophysical and biochemical properties of single bronchial smooth muscle cells from asthmatic subjects. Am J Physiol Lung Cell Mol Physiol 2002; 283: L1181L1189.

15. Bai TR. Abnormalities in airway smooth muscle in fatal asthma. A comparison between trachea and bronchus. Am Rev Respir Dis 1991; 143: 441-443.

16. Bjorck T, Gustafsson LE, Dahlen SE. Isolated bronchi from asthmatics are hyperresponsive to adenosine, which apparently acts indirectly by liberation of leukotrienes and histamine. Am Rev Respir Dis 1992; 145: 1087-1091.

17. Wagers S, Lundblad LK, Ekman M, Irvin CG, Bates JH. The allergic mouse model of asthma: normal smooth muscle in an abnormal lung? J Appl Physiol 2004; 96: 2019-2027.

18. Fredberg JJ. Airway narrowing in asthma: does speed kill? Am J Physiol Lung Cell Mol Physiol 2002; 283: L1179L1180.

19. Johnson PR, Roth M, Tamm M, Hughes M, Ge Q, King G, et al. Airway smooth muscle cell proliferation is increased in asthma. Am J Respir Crit Care Med 2001; 164: 474-477.

20. Cox G, Miller JD, McWilliams A, Fitzgerald JM, Lam S. Bronchial thermoplasty for asthma. Am J Respir Crit Care Med 2006; 173: 965-969.

21. Ishida K, Pare PD, Thomson RJ, Schellenberg RR. Increased in vitro responses of tracheal smooth muscle from hyperresponsive guinea pigs. J Appl Physiol 1990; 68: 13161320. 
22. Jiang H, Rao K, Liu X, Halayko AJ, Liu G, Stephens NL. Early changes in airway smooth muscle hyperresponsiveness. Can J Physiol Pharmacol 1994; 72: 1440-1447.

23. Blanc FX, Coirault C, Salmeron S, Chemla D, Lecarpentier $Y$. Mechanics and crossbridge kinetics of tracheal smooth muscle in two inbred rat strains. Eur Respir J 2003; 22: 227234.

24. Burgess JK, Johnson PR, Ge Q, Au WW, Poniris MH, McParland BE, et al. Expression of connective tissue growth factor in asthmatic airway smooth muscle cells. Am J Respir Crit Care Med 2003; 167: 71-77.

25. Chambers LS, Black JL, Ge Q, Carlin SM, Au WW, Poniris $M$, et al. PAR-2 activation, PGE2, and COX-2 in human asthmatic and nonasthmatic airway smooth muscle cells. Am J Physiol Lung Cell Mol Physiol 2003; 285: L619-L627.

26. Kapsali T, Permutt S, Laube B, Scichilone N, Togias A. Potent bronchoprotective effect of deep inspiration and its absence in asthma. J Appl Physiol 2000; 89: 711-720.

27. Fish JE, Peterman VI, Cugell DW. Effect of deep inspiration on airway conductance in subjects with allergic rhinitis and allergic asthma. J Allergy Clin Immunol 1977; 60: 41-46.

28. Lim TK, Pride NB, Ingram RH Jr. Effects of volume history during spontaneous and acutely induced air-flow obstruction in asthma. Am Rev Respir Dis 1987; 135: 591-596.

29. Lim TK, Ang SM, Rossing TH, Ingenito EP, Ingram RH Jr. The effects of deep inhalation on maximal expiratory flow during intensive treatment of spontaneous asthmatic episodes. Am Rev Respir Dis 1989; 140: 340-343.

30. Gunst SJ, Stropp JQ, Service J. Mechanical modulation of pressure-volume characteristics of contracted canine airways in vitro. J Appl Physiol 1990; 68: 2223-2229.

31. Fredberg JJ, Inouye DS, Mijailovich SM, Butler JP. Perturbed equilibrium of myosin binding in airway smooth muscle and its implications in bronchospasm. Am J Respir Crit Care Med 1999; 159: 959-967.

32. Lazaar AL, Panettieri RA Jr. Airway smooth muscle as a regulator of immune responses and bronchomotor tone. Clin Chest Med 2006; 27: 53-69, vi.

33. Global Strategy for Asthma Management and Prevention, Global Initiative for Asthma (GINA). Available at http:// www.ginasthma.org.

34. Muro S, Minshall EM, Hamid QA. The pathology of chronic asthma. Clin Chest Med 2000; 21: 225-244.

35. Hirst SJ. Regulation of airway smooth muscle cell immunomodulatory function: role in asthma. Respir Physiol Neurobiol 2003; 137: 309-326

36. Faffe DS, Whitehead T, Moore PE, Baraldo S, Flynt L, Bourgeois $\mathrm{K}$, et al. IL-13 and IL-4 promote TARC release in human airway smooth muscle cells: role of IL-4 receptor genotype. Am J Physiol Lung Cell Mol Physiol 2003; 285: L907-L914.

37. Leigh R, Ellis R, Wattie J, Southam DS, De Hoogh M, Gauldie J, et al. Dysfunction and remodeling of the mouse airway persist after resolution of acute allergen-induced airway inflammation. Am J Respir Cell Mol Biol 2002; 27: 526-535.

38. DiCosmo BF, Geba GP, Picarella D, Elias JA, Rankin JA, Stripp BR, et al. Airway epithelial cell expression of interleukin-6 in transgenic mice. Uncoupling of airway inflammation and bronchial hyperreactivity. J Clin Invest 1994; 94: 20282035 .
39. Zhu Z, Homer RJ, Wang Z, Chen Q, Geba GP, Wang J, et al. Pulmonary expression of interleukin-13 causes inflammation, mucus hypersecretion, subepithelial fibrosis, physiologic abnormalities, and eotaxin production. J Clin Invest 1999; 103: 779-788.

40. Homer RJ, Elias JA. Airway remodeling in asthma: therapeutic implications of mechanisms. Physiology 2005; 20: 28-35.

41. Busse W, Banks-Schlegel S, Noel P, Ortega H, Taggart V, Elias J. Future research directions in asthma: an NHLBI Working Group report. Am J Respir Crit Care Med 2004; 170: 683-690.

42. Faffe DS, Flynt L, Bourgeois K, Panettieri RA Jr, Shore SA. Interleukin-13 and interleukin-4 induce vascular endothelial growth factor release from airway smooth muscle cells: role of vascular endothelial growth factor genotype. Am J Respir Cell Mol Biol 2006; 34: 213-218.

43. Faffe DS, Flynt L, Mellema M, Whitehead TR, Bourgeois K, Panettieri RA Jr, et al. Oncostatin M causes VEGF release from human airway smooth muscle: synergy with IL-1beta. Am J Physiol Lung Cell Mol Physiol 2005; 288: L1040L1048.

44. Wills-Karp M, Luyimbazi J, Xu X, Schofield B, Neben TY, Karp CL, et al. Interleukin-13: central mediator of allergic asthma. Science 1998; 282: 2258-2261.

45. Johnson PR, Black JL, Carlin S, Ge Q, Underwood PA. The production of extracellular matrix proteins by human passively sensitized airway smooth-muscle cells in culture: the effect of beclomethasone. Am J Respir Crit Care Med 2000; 162: 2145-2151.

46. Knox AJ, Corbett L, Stocks J, Holland E, Zhu YM, Pang L. Human airway smooth muscle cells secrete vascular endothelial growth factor: up-regulation by bradykinin via a protein kinase $C$ and prostanoid-dependent mechanism. FASEB J 2001; 15: 2480-2488.

47. Covar RA, Spahn JD, Murphy JR, Szefler SJ. Progression of asthma measured by lung function in the childhood asthma management program. Am J Respir Crit Care Med 2004; 170: 234-241.

48. Davies DE, Wicks J, Powell RM, Puddicombe SM, Holgate ST. Airway remodeling in asthma: new insights. J Allergy Clin Immunol 2003; 111: 215-225.

49. Bucchieri F, Puddicombe SM, Lordan JL, Richter A, Buchanan D, Wilson SJ, et al. Asthmatic bronchial epithelium is more susceptible to oxidant-induced apoptosis. Am J Respir Cell Mol Biol 2002; 27: 179-185.

50. Trautmann A, Schmid-Grendelmeier P, Kruger K, Crameri R, Akdis M, Akkaya A, et al. T cells and eosinophils cooperate in the induction of bronchial epithelial cell apoptosis in asthma. J Allergy Clin Immunol 2002; 109: 329-337.

51. Puddicombe SM, Torres-Lozano C, Richter A, Bucchieri F, Lordan JL, Howarth $\mathrm{PH}$, et al. Increased expression of p21(waf) cyclin-dependent kinase inhibitor in asthmatic bronchial epithelium. Am J Respir Cell Mol Biol 2003; 28: 61-68.

52. Slade DJ, Kraft M. Airway remodeling from bench to bedside: current perspectives. Clin Chest Med 2006; 27: 71-85, vi.

53. Boxall C, Holgate ST, Davies DE. The contribution of transforming growth factor-beta and epidermal growth factor signalling to airway remodelling in chronic asthma. Eur Respir 
J 2006; 27: 208-229.

54. Kalluri R, Neilson EG. Epithelial-mesenchymal transition and its implications for fibrosis. J Clin Invest 2003; 112: 1776-1784.

55. Thiery JP, Sleeman JP. Complex networks orchestrate epithelial-mesenchymal transitions. Nat Rev Mol Cell Biol 2006; 7: $131-142$.

56. Warburton D, Schwarz M, Tefft D, Flores-Delgado G, Anderson KD, Cardoso WV. The molecular basis of lung morphogenesis. Mech Dev 2000; 92: 55-81.

57. Knight DA, Lane CL, Stick SM. Does aberrant activation of the epithelial-mesenchymal trophic unit play a key role in asthma or is it an unimportant sideshow? Curr Opin Pharmacol 2004; 4: 251-256.
58. Hashimoto S, Gon Y, Takeshita I, Matsumoto K, Maruoka $\mathrm{S}$, Horie $\mathrm{T}$. Transforming growth factor-beta1 induces phenotypic modulation of human lung fibroblasts to myofibroblast through a c-Jun-NH2-terminal kinase-dependent pathway. Am J Respir Crit Care Med 2001; 163: 152-157.

59. Iwano M, Plieth D, Danoff TM, Xue C, Okada H, Neilson EG. Evidence that fibroblasts derive from epithelium during tissue fibrosis. J Clin Invest 2002; 110: 341-350.

60. Demayo F, Minoo P, Plopper CG, Schuger L, Shannon J, Torday JS. Mesenchymal-epithelial interactions in lung development and repair: are modeling and remodeling the same process? Am J Physiol Lung Cell Mol Physiol 2002; 283: L510-L517. 